\title{
Correction to: Reliability, Construct and Predictive Validity of the Hong Kong Chinese Orebro Musculoskeletal Pain Screening Questionnaire
}

\author{
Raymond Chi Chung Tsang ${ }^{1}$. Jamie Sau Ying Lau ${ }^{2} \cdot$ Susane So Fong Kwong ${ }^{3} \cdot$ Eric Ming Loi So ${ }^{4}$. \\ Rainbow Ka Yee Law ${ }^{5}$. Thomas Fu Yan Wong ${ }^{6} \cdot$ Edwin Wai Chi Lee $^{7}$
}

Published online: 15 February 2018

(c) Springer Science+Business Media, LLC, part of Springer Nature 2018

\section{Correction to: Journal of Occupational Rehabilitation https://doi.org/10.1007/s10926-016-9689-3}

The original version of this article unfortunately contained a mistake. The given and family name of all the authors were incorrect. The author names have now been corrected.

The original article can be found online at https://doi.org/10.1007/ s10926-016-9689-3.

Raymond Chi Chung Tsang

tsangccr@ha.org.hk

1 Physiotherapy Department, MacLehose Medical Rehabilitation Centre, Hospital Authority, 7 Sha Wan Drive, Sandy Bay, Hong Kong, Hong Kong SAR, People's Republic of China

2 Physiotherapy Department, Prince of Wales Hospital, Hospital Authority, 30-32 Ngan Shing Street, Shatin, New Territories, Hong Kong, Hong Kong SAR, People's Republic of China

3 Physiotherapy Department, Tang Shiu Kin Hospital, Hospital Authority, 282 Queen's Road East, Wan Chai, Hong Kong, Hong Kong SAR, People's Republic of China

4 Physiotherapy Department, Princess Margaret Hospital, Hospital Authority, 2-10 Princess Margaret Hospital Road, Lai Chi Kok, Kowloon, Hong Kong, Hong Kong SAR, People's Republic of China
5 Department of Physical Medicine \& Rehabilitation, Hong Kong Sanatorium \& Hospital, 2 Village Road, Happy Valley, Hong Kong, Hong Kong SAR, People's Republic of China

6 Physiotherapy Department, Queen Elizabeth Hospital, Hospital Authority, 30 Gascoigne Road, Yau Ma Tei, Kowloon, Hong Kong, Hong Kong SAR, People's Republic of China

7 Occupation Medicine Care Service, New Territories East Cluster, Hospital Authority, 30-32 Ngan Shing Street, Shatin, New Territories, Hong Kong, Hong Kong SAR, People's Republic of China 